\title{
ROUNDUP360
}

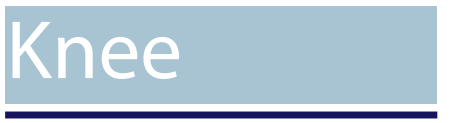

Anterior cruciate ligament

a The semitendinosus tendon is widely used as an anterior cruciate ligament $(A C L)$ graft. It is clearly desirable to maintain the morphology of the semitendinosus muscletendon complex after harvesting, if possible. Surgeons from Hiroshima (Japan) investigated this in 39 patients who underwent $A C L$ reconstruction with autologous semitendinosus tendon. After surgery the knee was immobilised for three days in 24 patients (control group) and for between 10 and 14 days in 15 patients (study group). Three-dimensional CT scanning was then undertaken at six and/or 12 months after surgery in all patients in order to identify any morphological changes in the semitendinosus muscle-tendon junction. Pleasingly, successful regeneration of the semitendinosus tendon was found in $38 / 39$ patients, although prolonged immobilisation (10 to 14 days) of the knee could not prevent morphological changes occurring in the semitendinosus muscle-tendon complex.' 360 concludes that one might as well encourage patients to mobilise early after $A C L$ reconstruction when a semitendinosus autograft is used.

- Computer-assisted surgery is not the sole domain of knee replacement. The technique may also be used for ligament reconstruction. Researchers from Rotterdam (The Netherlands) have investigated this through a number of major databases in order to compare the results for computer-assisted $\mathrm{ACL}$ and posterior cruciate ligament $(\mathrm{PCL})$ reconstruction surgery with conventional techniques. There were four randomised controlled trials included (266 participants) and some difficulties encountered because of poor reporting of trial methods. However, the authors considered that a clearly favourable effect of computer-assisted surgery could not be found in the field of cruciate-ligament reconstruction in the knee but that further studies were needed. ${ }^{2}$

- Techniques of arthroscopic meniscal repair continue to evolve with continuing debate about the advantages and disadvantages of inside-out versus all-inside repairs. However, results may be confounded by any associated ACL reconstruction or deficiency. Researchers from Ann Arbor (USA) thus undertook a systematic review of the problem using a number of different databases, including MEDLINE, EMBASE, Cochrane Database of Systematic Reviews

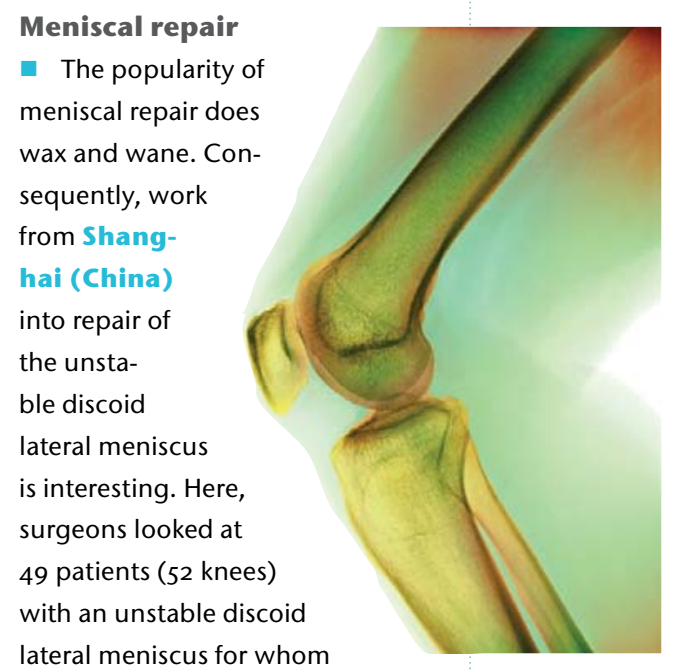

and others. They found 19 studies that included data specific to isolated meniscal tears, establishing a failure rate of $17 \%$ for the inside-out technique and $19 \%$ for all-inside. The message was simple and clear. Whichever technique is chosen, the results are likely to be similar. Any differences in an arthroscopic meniscectomy and suture of the peripheral rim had been performed. The patients were followed up for a mean of 20.8 months and assessed by means of MRI scan and Lysholm and HSS (Hospital for Special Surgery) outcome measures. Excellent-to-good results were found in nearly $88 \%$ of patients, suggesting that arthroscopic meniscectomy and suture of the peripheral rim is a good choice for this group of patients. ${ }^{3}$ healing rate after meniscal repair are probably down to the pattern of tear or the integrity of the $\mathrm{ACL}$ rather than the repair method selected. ${ }^{4}$

- Meanwhile researchers from St Louis (USA), again through a systematic literature review, have compared the rates of re-operation and clinical outcomes after partial meniscectomy and meniscal repair. A number of different repair techniques were studied - inside-out, outside-in, and all-inside. At short- and long-term follow-up, partial meniscectomy had a lower re-operation rate $(1.4 \%$ short term; $3.9 \%$ long term) than isolated meniscal repair ( $16.5 \%$ short term; $20.7 \%$ long term). Medial meniscal repairs had a higher re-operation rate than lateral repairs. However, partial lateral meniscectomies had a slightly higher re-operation rate than partial medial meniscectomies. Although meniscal repairs had a higher re-operation rate than partial meniscectomies, they were associated with better long-term outcomes. Despite this being a level IV study, it appears the authors have generally come out on the side of meniscal repair. ${ }^{5}$

\section{Anterior knee pain}

- Anterior knee pain has long been one of the great dilemmas of knee surgery with multiple procedures created to resolve it, many of which have had limited effect. Work on the role of in-shoe foot orthoses by teams from Brisbane and Canberra (Australia) thus strikes 360 as particularly interesting. The researchers took 40 patients with anterior knee pain in order to compare an in-shoe foot orthoses with a wait-and-see treatment policy over a six-week period. The orthoses easily won, with significant improvements in pain and function being observed. Furthermore, patients with greater midfoot mobility did best. $^{6}$

- Another continuing debate is whether or not to resurface the patella at total knee replacement and, for that matter, whether the 
design of components used might make a difference. Surgeons from Oswestry (UK) have looked at this with a meta-analysis of 7075 knee replacements, for which 3463 received a patellar resurfacing and 3612 did not. No significant differences were found between the two groups in the incidence of postoperative anterior knee pain, nor were there any differences created by prosthetic design. There was, however, a higher incidence of reoperation in patients who had not received a patellar resurfacing. ${ }^{7} 360$ agrees with the authors, that this increase may easily be attributed to the fact that a secondary patellar resurfacing adds a surgical option for the treatment of postreplacement anterior knee pain. This thereby artificially increases the rate of re-operation in the nonresurfacing group.

\section{Total knee replacement}

- Osteoarthritis and replacement of the knee are well known to cause deficiencies in proprioception.

Meanwhile proprioceptive training has become popular in athletes as it leads to economy of movement and energy saving. Workers from Innsbruck (Austria) have looked at the application of proprioceptive training in the field of knee replacement by providing six weeks of such training before knee replacement surgery. The training group was stood alongside controls that received knee replacement alone.
Both groups improved significantly after surgery but for those patients who received pre-operative proprioceptive training, there was an improvement in post-operative standing balance six weeks after the procedure. There was, however, no difference in clinical outcome seen between the two groups. ${ }^{8}$ So what about proprioceptive training? 360 feels the jury is still out.

- The accuracy of component alignment at total knee replacement is widely recognised as being important to long-term outcome. Computerassisted navigation can clearly help with this and, as a consequence, has been widely investigated. Researchers from Beijing (China) have looked at the role of computer-assisted navigation for a randomised, simultaneous bilateral knee replacement procedure in 32 patients. Computerassisted navigation was used in one knee and conventional replacement on the other. The patients and the follow-up surgeons were blinded as to which technique had been used. The results were clear. In every case, computer-assisted navigation allowed component coronal alignment within $3^{\circ}$ of the mechanical axis. This was significantly better than the alignment obtained with conventional total knee replacement. ${ }^{9}$

\section{Pulsed shortwave treatment for osteoarthritis \\ - To avoid knee replacement altogether must be a sensible ambi- tion, so methods of conservative}

treatment are well worth investigating. A team from a physical therapy department in São Paulo (Brazill) investigated the use of pulsed shortwave treatment in the management of osteoarthritis of the knee.

This has been widely used already but the dose and application time have not been well established. The study comprised four groups. There were 35 patients who received no shortwave at all and acted as controls. There were then 23 who received placebo treatment, 32 who received low-dose shortwave and 31 high-dose. Patients were followed up for 12 months. The results showed that both treatment groups had a significant reduction in pain, with improved function and that low-dose shortwave appeared to be more effective in the longer term. These results were achieved without physical exercise, which might also have positively influenced the results. ${ }^{10} 360$ concludes that there is clearly more to shortwave treatment than meets the eye, although the high dropout rate for this study means that the 12-month results need to be interpreted with caution.

\section{REFERENCES}

1. Nakamae A, Deie M, Adachi N, et al. Effects of knee immobilization on morphological changes in the semitendinosus muscle-tendon complex after hamstring harvesting for anterior cruciate ligament reconstruction: evaluation using threedimensional computed tomography. J Orthop Sci 2011;(Epub ahead of print).
2. Meuffels DE, Reijman M, Scholten RJ,

Verhaar JA. Computer assisted surgery for knee ligament reconstruction. Cochrane Database Syst Rev 2011;6:CDo07601.

3. Jiao S, Hongyun L, Shiyi C, et al. Clinical outcome of arthroscopic meniscectomy combined with suture for treatment of unstable discoid lateral meniscus. Chin JSports Med 2011;30:609-612. 4. Grant JA, Wilde J, Miller BS, Bedi A. Comparison of inside-out and all-inside techniques for the repair of isolated meniscal tears: a systematic review. Am JSports Med 2011;(Epub ahead of print). 5. Paxton ES, Stock MV, Brophy RH. Meniscal repair versus partial meniscectomy: a systematic review comparing re-operation rates and clinical outcomes. Arthroscopy 2011;27:1275-1288.

6. Mills $K$, Blanch $P$, Dev $P$, Marin M, Vicenzino B. A randomised control trial of short term efficacy of in-shoe foot orthoses compared with a wait and see policy for anterior knee pain and the role of foot mobility. Br JSports Med 2011; (Epub ahead of print).

7. Pavlou G, Meyer C, Leonidou A, et al. Patellar resurfacing in total knee arthroplasty: does design matter? A meta-analysis of 7075 cases. J Bone Joint Surg [Am] 2011;93-A:1301-1309.

8. Gstoettner M. Raschner C, Dirnberger E, Leimser H, Krismer M. Preoperative proprioceptive training in patients with total knee arthroplasty. Knee 2011;18:265-270.

9. Zhang GQ Chen JY, Chai W, Liu M, Wang Y. Comparison between computer-assisted-navigation and conventional total knee arthroplasties in patients undergoing simultaneous bilateral procedures: a randomized clinical trial. J Bone Joint Surg [Am] 2011;93-A:1190-1196.

10. Fukuda TY, Alves da Cunha R, Fukuda VO, et al. Pulsed shortwave treatment in women with knee osteoarthritis: a multicenter, randomized, placebo-controlled clinical trial. Phys Ther 2011;91:1009-1017. 